\title{
Passagem De Plantão Em Unidades De Terapia Intensiva Pediátrica: Interface Com A Segurança Do Paciente
}

\author{
Shift Changes In Pediatric Intensive Care Units: Interface With Patient Safety \\ Pasaje De Turno En Unidades De Terapia Intensiva Pediátrica: Interfaz Con La Seguridad Del \\ Paciente
}

Ingrid Mayara Almeida Valera ${ }^{1}$, João Lucas Campos de Oliveira $^{2}$, Ana Carolina Simões Pereira ${ }^{3}$, Andressa Martins Dias ${ }^{4}$ Maria do Carmo Fernandez Lourenço Haddad ${ }^{5}$, Laura Misue Matsuda ${ }^{6}$.

\section{Resumo}

Este estudo teve como objetivo identificar aspectos de afeto à segurança do paciente na passagem de plantão da equipe de enfermagem em Unidades de Terapia Intensivas Pediátricas. Adotou-se a metodologia de estudo transversal, descritivo e quantitativo. A coleta de dados deu-se por observação não participante das Passagens de Plantão (PP), durante sete dias consecutivos,

\footnotetext{
1 Mestra em Enfermagem. Enfermeira. União Oeste Paranaense de Estudos e Combate ao Câncer - UOPECCAN. Email: ingrid_imav@hotmail.com
${ }^{2}$ Doutor em Enfermagem. Professor-Adjunto. Departamento de Assistência e Orientação Profissional Escola de Enfermagem Universidade Federal do Rio Grande do Sul. E- mail: enfjoaolcampos@yahoo.com.br.

3 Mestra em Enfermagem. Professora Colaboradora. Universidade Estadual do Paraná - Unespar (Campus de Paranavaí). E-mail: carolsimoesenf@gmail.com.
${ }^{4}$ Mestra em Enfermagem. Professora Colaboradora. Curso de Graduação da Universidade Estadual de Maringá E-mail: andressam_dias@yahoo.com.br.

\footnotetext{
${ }^{5}$ Doutora em Enfermagem. Professora Sênior. Programa de Pós-Graduação em Enfermagem da Universidade Estadual de Londrina-PR. E-mail: carmohaddad@gmail.com

6 Pós-Doutora em Enfermagem. Professora Associada. Programa de Pós-Graduação em Enfermagem da Universidade Estadual de Maringá. E-mail: lauramisuem@gmail.com
}

nas Unidades de Terapia Intensivas Pediátricas de três hospitais universitários públicos do Paraná, Brasil. Utilizou-se o instrumento "Passagem de Plantão da Equipe de Enfermagem em Unidade de Cuidado Intensivo Neonatal", adaptado ao estudo. Aos dados tabulados, empregou-se análise estatística descritiva. Houve prevalência do método verbal $(75,9 \%)$, sem anotações escritas; não participação dos acompanhantes $(75,9 \%)$ e frequentes interrupções na passagem de plantão $(53,7 \%)$. A identificação do paciente; seu estado de saúde e evolução foram aspectos positivos à segurança do paciente nas PP. Embora apresentem espaço para melhoria, de modo geral, as PP mostraram-se consoantes ao preconizado.

Descritores: Comunicação. Troca de Informações. Segurança do Paciente. Terapia Intensiva. Enfermagem Pediátrica.

\section{Resumen}

Este estudio tuvo como objetivo identificar aspectos de afecto a la seguridad del paciente en el paso de turno del equipo de enfermería en Unidades de Terapia Intensivas 
Pediátricas. Estudio transversal, descriptivo y cuantitativo. La recolección de datos se dio por observación no participante de las Pasajens de Plantón (PP), durante siete días consecutivos en tres Unidades de Terapia Intensivas Pediátricas de hospitales universitarios de los públicos de Paraná, Brasil. Se utilizó el formulario Pasaje de Planta del Equipo de Enfermería en Unidad de Cuidado Intensivo Neonatal, adaptado al estudio. A los datos tabulados, se empleó análisis estadístico descriptivo. Hubo prevalencia del método verbal $(75,9 \%)$, sin anotaciones escritas; no participación de los acompañantes $\quad(75,9 \%) \quad y \quad$ frecuentes interrupciones en el paso de turno $(53,7 \%)$. La identificación del paciente; su estado de salud y evolución fueron aspectos positivos a la seguridad del paciente en las PP. Aunque presentamos espacio para la mejora, en general, las PP se mostraron consonantes al preconizado.

Descriptores: Comunicación. Intercambio de información. Seguridad del paciente. Terapia Intensiva. Enfermería Pediátrica.

\section{Abstract}

This study aimed to identify aspects of affection to the patient's safety in the passage of the nursing team on duty in Pediatric Intensive Care Units. Descriptive, quantitative and cross-sectional study. Data collection was done through non-participant observation of the Shift Changes (SC) for
}

seven consecutive days in three Pediatric Intensive Care Units of university hospitals in the state of Paraná, Brazil. The Nursing Team Nursing Team form was used in the Neonatal Intensive Care Unit, adapted to the study. Data were tabulated using descriptive statistical analysis. There was a prevalence of the verbal method $(75.9 \%)$, without written annotations; (75.9\%) and frequent interruptions in the shift (53.7\%). Patient identification; their health status and evolution were positive aspects to patient safety in PP. Although there is room for improvement, in general, the PP showed consonants to the recommended one.

Key words: Communication. Information exchange. Patient safety. Intensive therapy. Pediatric Nursing.

\section{Introdução}

$\mathrm{Na}$ saúde, o termo Qualidade é conceituado como um conjunto de cuidados eficazes, eficientes, acessíveis, aceitáveis, equitativos e seguros $^{(1)}$. Em virtude desta polissemia e complexidade, fatores relacionados à qualidade suscitam atenção de profissionais, gestores, usuários de saúde e sociedade em geral, que reconhecem a qualidade como instrumento-chave para a manutenção do potencial competitivo de qualquer empresa ${ }^{(2)}$.

Notadamente, na área da saúde, a qualidade dos serviços prestados se relaciona intimamente com a segurança do paciente ${ }^{(3)}$, 
que é definida como a redução do risco de dano desnecessário aos consumidores do cuidado $^{(4)}$. Por sua vez, a segurança do paciente é fortemente influenciada, dentre outros fatores, pela comunicação entre os profissionais que desempenham o trabalho assistencial e correlato ${ }^{(5)}$. Tendo tal premissa em vista, e diante do cenário de insegurança relacionada aos serviços de saúde, a Organização Mundial da Saúde (OMS), em parceria com a Joint Commission International (JCI), lançou a Aliança Mundial para a Segurança do Paciente, com o intuito de incentivar a implementação de seis Metas Internacionais de Segurança do Paciente, dentre as quais, melhorar a comunicação entre profissionais de saúde ${ }^{(6)}$.

Uma forma de comunicação com foco na continuidade e na segurança do cuidado é a passagem de plantão $(\mathrm{PP})^{(7)}$. Nessa modalidade comunicativa, os profissionais visam transmitir aos colegas que irão assumir o próximo turno de trabalho informações referentes aos pacientes, vivenciadas durante o período de atuação, de forma clara e objetiva $^{(7)}$. Portanto, a finalidade essencial da PP é viabilizar a continuidade do cuidado ${ }^{(7-8)}$.

A literatura aponta existência de falhas na comunicação, como no caso das PP, entre os cuidadores, principalmente enfermeiros, devido ao maior contato com os pacientes, a realizarem mais procedimentos, e por isso serem os principais responsáveis por erros na assistência que podem resultar em prejuízos irreversíveis $^{(5,8)}$.

Sabe-se que em hospitais, principalmente em unidades críticas, a PP se constitui como prática indispensável. A premissa anterior é ainda mais explícita, ao se considerar Unidades de Terapia Intensiva Pediátrica (UTI-P) que atendem, exclusivamente, clientes considerados vulneráveis, tanto pelo seu estado clínico de gravidade, como pela característica intrínseca à idade. Nesta circunstância, a obra ${ }^{(9)}$ intitulada "The art of effective hand offs: what is the evidence?", informa que as trocas de informações podem afetar a assistência de saúde aos clientes, principalmente em cuidados pediátricos e que, melhorias na comunicação das PP tendem à otimização dos resultados, minimização de erros e de desperdícios no cuidado.

Ante ao exposto, evidencia-se a necessidade de investigações sobre como ocorrem as PP em instituições de saúde. Estudos neste escopo podem servir de subsídio à tomada de decisão que confluam ao atendimento mais seguro, mediado pela comunicação eficaz.

Uma vez justificada a inclinação para investigar a problemática enunciada, o presente estudo se apoia no seguinte questionamento: Em relação à segurança do paciente, como se apresentam as PP da equipe de enfermagem em UTI-P? Logo, para responder à indagação, objetivou-se 
identificar aspectos de afeto à segurança do paciente na passagem de plantão da equipe de enfermagem em Unidades de Terapia Intensivas Pediátricas.

\section{Revisão de Literatura}

A área da saúde se diverge de outros segmentos de prestação de serviço, pois o trabalho produzido, que é complexo e intangível, é consumido concomitantemente a tal produção. Diante dessa complexidade, a comunicação na saúde é uma ferramenta indispensável ao processo de trabalho, expressando-se de diversas maneiras e por meio de diferentes instrumentos de comunicação $^{(10)}$.

A comunicação eficaz na saúde é vislumbrada como um aporte ao cuidado seguro, tal como falhas no processo comunicativo são reconhecidas como pontes para a insegurança na assistência ${ }^{(4)}$. Postula-se que o uso dos instrumentos ou meios que favorecem a comunicação em saúde, tanto no trabalho das equipes, como na relação profissional-usuário, precisa ser racional, além de adaptado à realidade. Porém, além do uso racional de instrumentos "comunicativos", sabe-se que a cultura organizacional ainda pode ser uma barreira para comunicação, interferindo na segurança do paciente, ao exemplo do medo da punição e da evidência do profissional como fatores limitantes na comunicação dos erros e da notificação de eventos adversos ${ }^{(6)}$.
No processo de trabalho em saúde, gama de meios e instrumentos disponíveis para comunicação é diversa, indo desde a comunicação verbal rotineira e comum ao trabalho eminentemente humano, até o emprego de estratégias acopladas de maior densidade tecnológica como, por exemplo, os sistemas de informação e os prontuários eletrônicos, já comuns atualmente ${ }^{(3)}$.

Ainda que o avanço tecnológico seja uma franca realidade evidenciada também na saúde, algumas práticas parecem ser persistentes, ao exemplo da Passagem de Plantão (PP), em especial na área hospitalar. Isso porque a finalidade primordial da PP é viabilizar a continuidade do cuidado, que, naturalmente neste ambiente, é divido entre turnos ininterruptos; ou ainda dar seguimento na assistência de um setor e/ou serviço a outro $^{(7)}$.

No processo de $\mathrm{PP}$, a comunicação eficaz é o cerne do seu propósito. Por sua vez, atua como uma ferramenta de humanização da assistência de saúde, a ser empregada pela equipe de trabalho, desde que os profissionais estejam motivados a reconher as várias configurações que a comunicação pode assumir, compreendendo que o paciente/familiar integra o processo de cuidar $^{(10)}$. A comunicação integra, inclusive, os momentos de PP, atividade inerente ao trabalho de enfermagem ${ }^{(11)}$.

Em que pese à importância da PP, a atividade envolve um momento de troca de 
informações entre os turnos das equipes de enfermagem, ofertando um espaço valioso para o informe da evolução do quadro de saúde do paciente; pendências no processo de trabalho e funcionamento da unidade; além de outros encaminhamentos ocorridos durante o plantão $^{(11)}$. Em hospitais, tal ferramenta possibilita a continuidade da assistência nas 24 horas de oferta de cuidado, permitindo a organização e planejamento de enfermagem, de modo a favorecer a segurança e a qualidade da assistência, intermediadas pela continuidade do cuidado ${ }^{(11-13)}$.

Visando o cuidado continuado, a PP apresenta diferentes modalidades de operacionalização, entre elas $^{(9,14)}$ :

- Verbal de maneira hierarquizada, quando os técnicos de enfermagem passam as informações para os técnicos do próximo turno e o mesmo ocorre entre os enfermeiros, de maneira isolada;

- Verbal em grupos, quando a passagem ocorre com a reunião de enfermeiros e técnicos de enfermagem, geralmente no posto de enfermagem;

- Em subgrupos;

- Em equipes multiprofissionais;

- À beira leito;

- Através de livro de plantão (relatório escrito); ou ainda

- Associando a passagem verbal e escrita.
Independente da modalidade empregada, todas as PP visam proporcionar a continuidade da assistência, adaptando-se à dinâmica de trabalho de cada serviço ${ }^{(11)}$.

\section{Método}

Este estudo se caracteriza como transversal, descritivo e de abordagem quantitativa. Foi realizado por meio de observação não participante sistemática das PP da equipe de enfermagem atuantes em UTI-P de três hospitais universitários públicos do Paraná, Brasil, durante o período de maio e dezembro de 2015. As unidades foram identificadas aleatoriamente como UTI-P I, II e III.

Ressalta-se que as observações das PP, nos três turnos de trabalho, foram realizadas após a anuência dos profissionais, através de assinatura do Termo de Consentimento Livre e Esclarecido (TCLE). As observações ocorreram durante sete dias ininterruptos. Assim, trata-se de um censo das trocas de plantão no recorte temporal proposto.

A UTI-P I possuía seis leitos, e a equipe de enfermagem era constituída por 11 enfermeiros e 10 técnicos de enfermagem. A UTI-P II era composta por cinco leitos e contava com seis enfermeiros, sete técnicos de enfermagem e, nove auxiliares de enfermagem. A UTI-P III possuía 10 leitos, sendo quatro para cuidados semi-intensivos, que no momento da coleta de dados estavam inativos, e seis para cuidados intensivos, 
considerados na pesquisa. Nesta unidade, o quadro da enfermagem era composto de seis enfermeiros, nove técnicos de enfermagem e 14 auxiliares de enfermagem.

Foram incluídas no estudo as PP realizadas por profissionais de enfermagem, constituídos por enfermeiros, técnicos e auxiliares de enfermagem com atuação no setor há pelo menos 60 dias. Os profissionais afastados do trabalho por qualquer motivo não foram considerados. Foram excluídas as PP nas quais um ou mais profissionais envolvidos não aceitaram participar. Desse modo, na UTI-P I, foram observadas PP de nove enfermeiros e sete técnicos de enfermagem, somando 16 participantes (uma recusa); na UTI-P II foram seis enfermeiros, sete técnicos e oito auxiliares de enfermagem, totalizando 21 profissionais (uma recusa) e; na UTI-P III, foram cinco enfermeiros, nove técnicos e 10 auxiliares, ou seja, 24 participantes (também com uma recusa).

O instrumento de coleta de dados consistiu um formulário de observação intitulado de "Passagem de Plantão da Equipe de Enfermagem em Unidade de Cuidado Intensivo Neonatal"(14), adaptado para os fins deste estudo. Originalmente o referido formulário caracteriza-se como um checklist e abrange informações referentes a dois grandes eixos: Identificação da PP e Questões relacionadas à $\mathrm{PP}$, cujas respostas assinaladas eram do tipo dicotômicas (Sim/Não), assinaladas por meio de "X". Para este estudo, o formulário foi dividido nas sessões: Passagem de plantão geral, para observação geral das passagens por turnos; e Passagem de plantão por paciente, para observação das trocas de informações de cada paciente, individualmente. Neste bloco de análise, observaram-se os seguintes aspectos de afeto à segurança do paciente direta ou indiretamente: identificação do paciente; estado de saúde; evolução; medicamentos utilizados; exames e procedimentos realizados; e assuntos administrativos.

As observações foram realizadas por três pesquisadores distintos, todos enfermeiros cursando pós-graduação stricto senso (Mestrado ou Doutorado), com treinamento conjunto prévio para padronização da coleta de dados. Munidos do formulário de coleta de dados e telefone celular para cronometragem das $\mathrm{PP}$, os pesquisadores se apresentavam às UTI-P em torno de 20 minutos anteriores ao término de cada plantão. Após contato com os profissionais, seguia-se às observações sistemáticas respeitando a extração das variáveis descritas no formulário, durante os sete dias consecutivos em cada unidade.

Os resultados foram sumarizados em planilhas eletrônicas do Microsoft Office Excel $^{\circledR}$, versão 2010. Após tabulação, procedeu-se a análise estatística descritiva, com medidas de proporção com auxílio do mesmo aparato tecnológico. Por fim, os achados dispostos em tabelas foram 
discutidos com base na literatura alusiva aos temas comunicação na PP e segurança do paciente.

Cumpre ressaltar que o estudo obedeceu integralmente aos princípios éticos que regem as pesquisas com seres humanos e foi aprovado pelo Comitê Permanente de Ética em Pesquisa Envolvendo Seres Humanos da Universidade Estadual de Maringá (COPEP/UEM), CAAE $\mathrm{n}^{\circ}$ 32206414.6.1001.0104 e Parecer no 866.802, de 2014.

\section{Resultados}

Das 63 PP gerais que ocorreram, foram observadas 54 nas três unidades pesquisadas, sendo, destas, $19(35,2 \%)$ na UTI-P I, 18 (33,3\%) na UTI-P II e 17 (31,5\%) na UTI-P
III. As nove trocas de turnos não analisadas foram "perdidas" por motivo de recusa de algum profissional. Do total das PP analisadas, $37(68,5 \%)$ foram realizadas em dias úteis, $13(24,1 \%)$ em finais de semana e quatro $(7,4 \%)$ em feriados. Constatou-se em todas $(100 \%)$ as PP o uso de linguagem verbal clara, audível e oportunidades para o esclarecimento de dúvidas. Não se observou em nenhuma PP o uso de qualquer aparato tecnológico, como gravadores de áudio, tablets, smartphones e computadores. $\mathrm{Na}$ Tabela 1 constam as características das PP gerais observadas.

Tabela 1 - Características das passagens de plantão gerais $(n=54)$ observadas em Unidades de Terapia Intensiva Pediátrica do Paraná, Brasil, 2015.

\begin{tabular}{lcccccccc}
\hline PP* Geral & \multicolumn{2}{c}{ UTI-P I } & \multicolumn{2}{c}{ UTI-P II } & \multicolumn{2}{c}{ UTI-P III } & \multicolumn{2}{c}{ Total } \\
Características & $\mathbf{n}$ & $\mathbf{\%}$ & $\mathbf{n}$ & $\boldsymbol{\%}$ & $\mathbf{n}$ & $\boldsymbol{\%}$ & $\mathbf{n}$ & $\%$ \\
\hline Local & & & & & & & & \\
Corredor da Unidade & - & - & - & - & 04 & 23,5 & 04 & 7,4 \\
Ao lado do leito do paciente & 19 & 100 & 18 & 100 & 13 & 76,5 & 50 & 92,6 \\
Duração (minutos) & & & & & & & & \\
$\leq 5$ & - & - & - & - & 04 & 23,5 & 04 & 7,4 \\
$6-10$ & 02 & 10,5 & 05 & 27,8 & 11 & 64,7 & 18 & 33,3 \\
$11-20$ & 13 & 68,4 & 13 & 72,2 & 02 & 11,8 & 28 & 51,9 \\
$21-30$ & 04 & 21,1 & - & - & - & - & 04 & 7,4 \\
Método & & & & & & & & \\
Verbal & 08 & 42,1 & 18 & 100 & 15 & 88,2 & 41 & 75,9
\end{tabular}


Verbal com anotações escritas

Participação de acompanhantes

Sim

Não

Interrupções

Conversas paralelas dos profissionais

Chegadas tardias (atrasos)

Intercorrências

Acompanhantes com dúvidas

Ruídos dos aparelhos da Unidade

\begin{tabular}{cccccccc}
- & - & 13 & 72,2 & - & - & 13 & 24,1 \\
19 & 100 & 05 & 27,8 & 17 & 100 & 41 & 75,9 \\
18 & 94,7 & 09 & 50 & 02 & 11,8 & 29 & 53,7 \\
04 & 22,2 & 01 & 11,1 & 02 & 100 & 7 & 24,1 \\
18 & 100 & 01 & 11,1 & - & - & 19 & 65,5 \\
04 & 22,2 & 01 & 11,1 & - & - & 05 & 17,2 \\
- & - & 01 & 11,1 & - & - & 01 & 3,4 \\
- & - & 09 & 100 & - & - & 09 & 31 \\
\hline
\end{tabular}

*PP Passagem de Plantão

Foram observadas 204 (100\%) PP individuais, ou seja, por paciente. Destas, 79 $(38,7 \%)$ ocorreram na UTI-P I; $73(35,8 \%)$ na
UTI-P II e; 52 (25,5\%) na UTI-P III. A saber, as informações repassadas durante as PP por paciente constam na Tabela 2.

Tabela 2 - Informações repassadas em passagens de plantão por paciente $(n=204)$ em

Unidades de Terapia Intensiva Pediátrica do Paraná, Brasil, 2015.

\begin{tabular}{lcccccccc}
\hline \multirow{2}{*}{$\begin{array}{l}\text { PP* por Paciente } \\
\text { Informações repassadas }\end{array}$} & \multicolumn{2}{c}{ UTI-P I } & \multicolumn{2}{c}{ UTI-P II } & \multicolumn{2}{c}{ UTI-P III } & \multicolumn{2}{c}{ Total } \\
\hline Identificação & $\mathbf{n}$ & $\boldsymbol{\%}$ & $\mathbf{n}$ & $\mathbf{\%}$ & $\mathbf{n}$ & $\boldsymbol{\%}$ & $\mathbf{n}$ & $\%$ \\
Pelo problema de saúde & 66 & 83,5 & 72 & 98,6 & 35 & 67,3 & 173 & 84,8 \\
Pelo primeiro nome & 02 & 3,1 & 26 & 36,1 & - & - & 28 & 16,1 \\
Pelo número do leito & 62 & 93,9 & 69 & 95,8 & 33 & 94,2 & 164 & 94,7 \\
Pelo nome da mãe & 01 & 1,5 & - & - & 02 & 5,7 & 03 & 1,7 \\
Paciente não é identificado & 01 & 1,5 & - & - & - & - & 01 & 0,5 \\
Estado de saúde & 13 & 16,5 & 01 & 1,4 & 17 & 32,7 & 31 & 15,2 \\
Alterações de sinais e sintomas & 75 & 94,9 & 73 & 100 & 51 & 98,1 & 199 & 97,5 \\
Alterações no exame físico & 62 & 82,6 & 72 & 98,6 & 51 & 100 & 185 & 92,9 \\
Evolução do quadro clínico & 52 & 69,3 & 72 & 98,6 & 36 & 70,5 & 160 & 80,4 \\
Evolução & 75 & 100 & 73 & 100 & 41 & 80,4 & 189 & 94,9 \\
Medicamentos utilizados & 79 & 100 & 73 & 100 & 44 & 84,6 & 196 & 96,1 \\
Horário & 69 & 87,3 & 46 & 63 & 35 & 67,3 & 150 & 73,5 \\
Via & 38 & 55,1 & 33 & 71,7 & 24 & 68,5 & 95 & 63,3 \\
& 19 & 27,5 & 23 & 50 & 15 & 46,8 & 57 & 38
\end{tabular}


Dose

Reações adversas

Medicamentos não administrados

Procedimentos realizados

Exames

Tipo

Horário

Cuidados

Resultados

Resultados relevantes**

Acompanhamento de familiar durante exame $\begin{array}{llllllll}53 & 76,8 & 24 & 52,2 & 16 & 45,7 & 93 & 62\end{array}$

$$
16
$$

23,9

$-$

-
$-\quad 19$

$\begin{array}{lll}6 & 23,2 \quad 05\end{array}$

$10,8 \quad 04$

$11,4 \quad 25$

16,6

$82,2 \quad 67$

$91,8 \quad 48$

$92,3 \quad 180$

88,2

$37,9 \quad 16$

$21,9 \quad 02$

$3,8 \quad 48$

23,5

$93,3 \quad 16$

$100 \quad 02$

$100 \quad 46$

95,8

$40 \quad 08$

50

$\begin{array}{lll}02 & 100 \quad 22\end{array}$

45,8

$30 \quad 01$

6,2

10

20,8

$10 \quad 33,3-07$

62,5

22

45,8

$10-33,3=07$

43,7

17

35,4

$\begin{array}{lll}01 & 3,3 & 01\end{array}$

6,2

02

4,1

Assuntos administrativos

$\begin{array}{llllllll}13 & 16,4 & 15 & 20,5 & 12 & 23,1 & 40 & 19,6\end{array}$

*PP Passagem de Plantão. **Resultados relevantes Resultados de exames recentes, no sentido de serem relevantes ao cuidado a ser continuado.

\section{Discussão}

Em relação à PP geral, observou-se o predomínio de linguagem verbal clara, audível e oportunidades para o esclarecimento de dúvidas entre os profissionais de enfermagem durante todas as trocas de turno. Isso possivelmente favorece a interação e a melhor comunicação, e, portanto, contribui à segurança do paciente.

A literatura ${ }^{(10)}$ aponta que todo processo comunicativo tem como propósito a interação entre os envolvidos, pois quando interagimos buscamos a interpretação dos fatos e de suas consequências, determinando significado aos relatos que transmitimos e recebemos.

$\mathrm{Na}$ saúde, a integração dos profissionais, sobretudo enfermeiros, advinda da comunicação adequada e, portanto, sem falhas produzidas por mal entendidos, auxilia Rev. Gestão \& Saúde (Brasília) Vol. 10, n. 03, Set. 2019. na transmissão de informações, na prestação de cuidados aos pacientes e na prevenção de danos $^{(10,15)}$, contribuindo para a segurança das ações desenvolvidas.

Ao considerar a necessidade de prevenção de danos aos pacientes, provocados por problemas de comunicação, especialmente nos tempos de avanços tecnológicos hodiernos, a tecnologia pode - e deve - ser empregada na melhoria da segurança, inclusive durante as PP, no sentido de confirmar, reforçar e/ou padronizar as informações que deverão ser transmitidas ${ }^{(11)}$.

Em contraponto à assertiva anterior, o que se constatou no estudo é que mesmo com a gama de ferramentas disponíveis, em nenhuma das UTI-P investigadas identificouse a utilização de qualquer instrumento tecnológico do tipo gravadores de áudio, 
tablets, smartphones ou computadores nas trocas de turno, o que pode evidenciar certa carência de recursos, desvalorização deste procedimento pelos profissionais que a realizam ou por parte das instituições.

Outro aspecto importante para a PP diz respeito ao local no qual esta ocorre. Este estudo revelou que, nas UTI-P investigadas, a maioria se deu ao lado do leito do paciente. Acredita-se que a preferência pela proximidade com o paciente durante a PP tenha ocorrido em função dos profissionais promoverem contato e contextualizar as informações, contribuindo para práticas mais seguras. Por outro lado, admite-se que esta escolha pode ter se dado em razão das características da própria unidade, a qual possui número reduzido de leitos, em comparação com outros setores e isso, conforme consta na literatura $^{(12)}$, facilita que a PP ocorra à beira do leito.

O local de realização da PP coaduna com os resultados de pesquisa conduzida com 707 profissionais de saúde australianos, destes sendo $60 \%$ enfermeiros, os quais confirmaram que a PP era realizada à beira do leito, por considerarem mais eficaz, em comparação àquela realizada em local distante do paciente ${ }^{(16)}$. Neste escopo, aludese que esta vantagem de contato próximo ao paciente para a PP é valiosa à segurança do paciente, já que o momento de troca de informações pode servir como oportunidade de aprendizado no trabalho; humanização do cuidado; e facilidade para recordar "pendências" assistenciais relacionadas à individualidade de cada paciente.

Ao considerar o tempo despendido para a realização da PP nas UTI-P observadas, a maioria dos profissionais de enfermagem realizou entre 11 e 20 minutos, dependendo do número de pacientes internados, das informações repassadas e das interferências ocorridas durante a comunicação.

Mesmo considerando que a menor UTIP possuía cinco leitos, o tempo mínimo de 11 minutos parece ser pouco porque, sabe-se à prática clínica, nesse setor o número de cuidados de enfermagem é elevado e a assistência dispensada usualmente é complexa. Estudo ${ }^{(7)}$ indica que o tempo gasto durante a PP se apresenta como um desafio para seu sucesso, pois pode influenciar de modo favorável ou desfavorável a continuidade do trabalho.

Segundo os referidos autores, se a PP for longa/extensa, tornará a atividade cansativa e poderá conduzir a equipe à desatenção. E, se for demasiadamente curta, a rapidez na entrega das informações poderá dar margem a perdas ou más interpretações que podem favorecer condutas errôneas e, com isso, interferir na segurança do paciente $^{(7)}$.

A cada troca de turno, a PP demanda comprometimento, tempo e preparo dos profissionais, uma vez que sua duração não limita-se exclusivamente à troca de 
informações, mas também à elaboração de notas prévias, registros e uma análise crítica final dos dados relevantes a serem comunicados $^{(7)}$.

Mais um fator que pode influenciar drasticamente a qualidade da comunicação nas PP e, consequentemente, como já se explorou anteriormente, a própria segurança do paciente, é o método adotado. Neste estudo, identificou-se que grande parte dos profissionais de enfermagem das UTI-P, optou pelo método verbal (fala) para passar as informações aos colegas e a minoria apoiouse em anotações. Estes dados contrapõem-se aos resultados de estudo realizado na Austrália, em que $73 \%$ dos profissionais relataram fazer uso de notas escritas e apenas $33 \%$ afirmaram confiar na memória ao comunicar as informações dos pacientes ${ }^{(16)}$.

É preocupante que a maioria dos profissionais de enfermagem das UTI-P investigadas dê preferência à transmissão exclusiva de informações "guardadas" na memória, que podem ser falhas e/ou incompletas, podendo comprometer a continuidade e a segurança da assistência prestada às crianças. Por esses motivos e, sustentando-se na literatura ${ }^{(16)}$, é fortemente incentivado que a PP verbal seja enriquecida com informações escritas e relevantes sobre os pacientes, para minimzar perdas de informações e erros de condutas. Já é amplamente conhecido que a clareza das informações escritas com a finalidade de complementar ou instituir o método da PP não devem substituir a completude e qualidade dos registros no prontuário do paciente, que têm valor ético-legal e constituem uma importante forma de comunicação no processo de trabalho da equipe de saúde.

Sobre a participação do acompanhante, destaca-se que, no geral, estes não são envolvidos no processo de PP. Observou-se inclusive que, apesar disso não contemplar o propósito da investigação, em muitos momentos durante a coleta de dados, os profissionais solicitavam aos familiares das crianças que se ausentassem da unidade enquanto as PP fossem realizadas.

Ao ponderar sobre o dado anterior, alvitra-se que é preciso refletir sobre a importância da participação do acompanhante na PP, sobretudo em unidades críticas, permitindo que ele(a) inclua informações que possam contribuir no cuidado da criança. Neste contexto, a literatura ${ }^{(16-17)}$ indica que a participação dos familiares na PP precisa ser encorajada pela equipe, pois esta prática pode tornar a comunicação mais eficaz, pois, a liberdade para comentar sobre os cuidados aos pacientes, bem como suas próprias preocupações e anseios, pode contribuir para a identificação de lacunas na comunicação e; também, prevenir possíveis eventos adversos.

Um dos principais fatores dificultadores e que contribui para a ineficácia das PP é a interrupção, visualizada em todas as UTI-P. 
Na UTI-P I, cerca de 95\% das PP sofreram interrupções e, dentre as interrupções de todas as UTI, os principais motivos foram: atrasos de profissionais, disparos de alarmes (ruídos) de aparelhos, conversas paralelas entre os membros da equipe, intercorrências com pacientes e, interrupções de acompanhantes para solicitar esclarecimentos sobre o estado de saúde do paciente.

A ocorrência de interrupções durante a PP, seja por pacientes, familiares e/ou outros profissionais, é um fato comum que reflete negativamente na continuidade do atendimento oferecido ${ }^{(13)}$, pois pode induzir à desatenção dos profissionais, comprometendo as informações transmitidas ou recebidas. No entanto, destaca-se que na prática assistencial tais interrupções sejam de difícil controle/intervenção, visto que por vezes os profissionais de enfermagem ocupam posição central no cerne da comunicação multiprofissional com pacientes e familiares.

Ainda sobre as interrupções, consideradas como pontos fracos da PP, os resultados obtidos a partir das observações das UTI-P, corroboram estudos ${ }^{(17,13)}$ desenvolvidos em diferentes setores hospitalares, nos quais as interrupções são atribuídas à falta de planejamento e à resistência dos profissionais em reconhecer a importância da PP para a qualidade e segurança do cuidado de enfermagem.

Por outro lado, a literatura ${ }^{(13)}$ aponta que as interrupções durante a PP, seja por usuários, acompanhantes, colegas ou serviços de apoio, são aspectos comuns, pois espelham o modelo de atenção hospitalar, que é dinâmico e contínuo. Por esta razão, a participação dos outros atores que completam a equipe de cuidados, ainda por meio de interrupções, pode ser um fator positivo, desde que as informações sejam úteis à continuidade da assistência. Desse modo, o fato das PP nas UTI-P serem exclusivas da equipe de enfermagem, muitas vezes em segregação hierárquica, talvez possa ser revisto, já que o número reduzido de leitos e a presença da equipe médica diuturnamente (por se tratar de um setor crítico) possivelmente favoreçam a PP da enfermagem em conjunto, ao menos, com profissionais médicos.

Em relação ao tipo de informação, é essencial que haja uma padronização dos dados a serem transmitidos, referentes à identificação do paciente, às suas condições; aos medicamentos utilizados; resultados de exames; procedimentos realizados; evolução do quadro clínico; recomendações e; no caso de pacientes pediátricos, qual familiar acompanhou a criança durante os cuidados ${ }^{(7)}$.

Apesar de os quesitos citados serem essenciais, autores $^{(11)}$ afirmam que a PP deve sempre oferecer informações críticas, as quais sejam importantes à transição segura do cuidado, independentemente do método ou estratégia de comunicação adotada.

Neste estudo, foi constatado que 15,2\% 
das crianças não foram identificadas pelos profissionais de enfermagem durante a passagem de informações a seus pares; $26,5 \%$ das PP não tiveram informações relacionadas a medicamentos utilizados durante o turno e em $76,5 \%$, os exames realizados não foram mencionados. Visualiza-se, a partir desses resultados, a omissão de informações consideradas essenciais a uma adequada PP e, consequentemente à segurança do paciente, pois o não repasse desses dados pode prejudicar o cuidado.

Dada à indispensabilidade e à diversidade de informações que necessitam ser transmitidas nas PP, a literatura ${ }^{(16)}$ propõe e disponibiliza vários métodos e protocolos padronizados de orientação da comunicação, que auxiliam os profissionais na execução da troca de turno, dentre os quais se destaca o mnemônico SBAR.

O método SBAR na PP da enfermagem tem por finalidade estruturar a comunicação de maneira organizada, clara e objetiva, obedecendo quatro itens representados por: $\mathrm{S}$ (Situation) - relato preciso da situação atual do paciente; B (Background) - informações pertinentes à sua história prévia; A (Assessment) - quadro clínico com dados reais que apoiem a tomada de decisão e; $R$ (Recommendation) - recomendações do enfermeiro à equipe de enfermagem, segundo as necessidades identificadas de cada paciente $^{(18)}$. Interpretando o método SBAR à luz dos resultados obtidos, percebe-se que, mesmo sem a utilização sistemática do método em questão, os profissionais demonstram cumprimento principalmente no que tange à situation (interpretada como estado de saúde); e assessment, se interpretada como a evolução do paciente.

Observa-se que, durante a PP, as informações transmitidas normalmente evidenciam características relacionadas à assistência direta aos pacientes, bem como aspectos gerenciais e de funcionamento da unidade, propiciando um olhar amplo do setor, otimizando o planejamento e organização das atividades ${ }^{(7)}$. Neste contexto, observou-se, neste estudo, que dados psicológicos ou mesmo religiosos dos pacientes, não foram abordados ou transmitidos, e isso é um exemplo da desvalorização dessas dimensões por parte dos profissionais.

Como sugestões para promover melhorias nas PP da equipe de enfermagem indicam-se ações de sensibilização, capacitação e monitoramento contínuo dos profissionais, para que a PP seja incorporada como ferramenta essencial à qualidade e segurança do cuidado, mediada pela comunicação eficaz.

\section{Conclusão}

Conclui-se que as PP da equipe de enfermagem nas unidades críticas pediátricas apresentaram aspectos que podem comprometer a segurança no atendimento pela comunicação eficaz, com destaque para: 
a adoção do método exclusivamente verbal, a falta de participação dos acompanhantes, o número expressivo de interferências e a omissão de dados em alguns casos, como: identificação do paciente, informações relacionadas a medicamentos e exames realizados. Apesar disso, de modo geral, ao analisar a totalidade os resultados apresentados, conjectura-se que as PP foram adequadas ao que se preconiza na literatura, embora exista espaço para melhorias.

O estudo apresenta como principal limitação a investigação de PP apenas de equipes de enfermagem em UTI-P. Portanto, indica-se a realização de pesquisas que abrangem a comunicação junto às demais categorias que constituem os grupos multidisciplinares atuantes em todos os setores dos serviços de saúde e, que tenham como foco a comunicação efetiva nas PP e suas implicações ao cuidado seguro. Este estudo contribui à militância para melhoria da comunicação em saúde e reforça a importância e a necessidade de valorização da PP, como forma de comunicação que auxilia na continuidade do cuidado e na segurança dos pacientes.

\section{Referências}

1. World Health Organization (WHO).Quality of care: a process for making strategic choices in health systems. Geneva; 2006.

2. Souza IG, Almeida AFS, Jesus VS, Siqueira SMC. The Benefits of Hospital Accreditation for Nursing Staff. Rev Bras Saud Func. [periódico 
na internet].2016 [citado 2017 dez. 18];1(11):07-15. Disponível em: http://www.seeradventista.com.br/ojs/i ndex.php/RBSF/article/view/700

3. Padilha EF, Haddad MCFL, Matsuda LM. Quality of nursing records in intensive care: evaluation through a retrospective audit. Cogitare Enferm. [periódico na internet]. 2014 [citado 2017 dez. 18];19(2):239-45. Disponível em: http://revistas.ufpr.br/cogitare/article/v iewFile/32103/22727

4. World Health Organization (WHO). More than words. Conceptual framework for the International Classification for Patient Safety (ICPS) - Final technical report. Geneva; 2009.

5. Ammouri AA, Tailakh AK, Muliira JK, Geethakrishnan R, Kindi A. Patient safety culture among nurses. Int Nurs Rev. [periódico na internet]. 2015 [citado 2017 dez. 18];62(1):10210. Disponível em: https://www.ncbi.nlm.nih.gov/pubmed $\underline{125495946}$

6. Moura GMSS, Magalhães AMM. Eventos Adversos relacionados à assistência em serviços de saúde: principais tipos. In: Anvisa. Agência Nacional de Vigilância Sanitária. Assistência segura: Uma reflexão teórica aplicada à prática. Brasília: 2013. p.65-75

7. Silva MF, Anders JC, Rocha PK, Souza AIJ, Burciaga VB. Communication in nursing shit handover: pediatric patient safety. Texto Contexto Enferm. [periódico na internet]. 2016 [citado 2017 dez. 18]; 25(3):e3600015. Disponível em: http://www.scielo.br/scielo.php?pid=S $\underline{010407072016000300322 \& \text { script }=\text { sci }}$ _abstract

8. Van Bogaert $\mathrm{P}, \quad$ Timmermans O, Weeks SM, Van Heusden D, Wouters K, Franck E. Nursing unit teams matter: Impact of unit-level nurse practice environment, nurse work characteristics, and burnout on nurse reported job outcomes, and quality of care, and patient adverse events--a cross-sectional survey. Int $\mathbf{J}$ Nurs Stud. [periódico na internet]. 2014 [citado 2017 dez. 18];51(8):1123-34. Disponível em: https://www.ncbi.nlm.nih.gov/pubme/ $\underline{24444772}$

9. Gephart SM. The art of effective hand offs: what is the evidence? Adv Neonatal Care. [periódico na internet]. 2012 [citado 2017 dez. 18];12(1):37-9. Disponível em: https://www.ncbi.nlm.nih.gov/pmc/art icles/PMC3272252/ 
10. Broca PV, Ferreira MA. Equipe de enfermagem e comunicação: contribuições para o cuidado de enfermagem. Rev Bras Enferm. [periódico na internet]. 2012 [citado 2017 dez. 20]; 65(1):97-103. Disponível em: http://www.scielo.br/pdf/reben/v65n1 14.pdf

11. Oliveira MC, Rocha RGM. Reflexão acerca da passagem de plantão: implicações na continuidade da assistência de enfermagem. Enferm. Rev. [periódico na internet]. 2016 [citado 2017 dez. 20];19(2):191-8. Disponível em: http://periodicos.pucminas.br/index.ph p/enfermagemrevista/article/view/131 $\underline{54}$

12. Llapa-Rodriguez EO, Oliveira CS, França TRS, Andrade JS, Campos MPA, Silva FJCP. Programación del cambio de turno desde la óptica de los profesionales de enfermería. Enferm. glob. [periódico na internet]. 2013 [citado 2017 dez. 20];12(31):206-31 Disponível em: http://scielo.isciii.es/scielo.php?script =sci_arttext\&pid=S1695$\underline{61412013000300013}$

13. Barbosa PMK, Barbosa VBA, Sores FV, Sales PRS, Barbosa FK, Silva LCP. Organização do processo de trabalho para passagem de plantão utilizando escore para dependência e risco clínico. RAS. 2013;15(58):1926.

14. Gonçalves MI, Rocha PK, Anders JC, Kusahara DM, Tomazoni A. Communication and patient safety in the chance-of-shift nursing report in neonatal intensive care units. Texto Contexto Enferm. [periódico na internet]. 2016 [citado 2017 dez. 20];25(1):e2310014. Disponível em: http://www.scielo.br/scielo.php?script $=$ sci_arttext\&pid=S010407072016000 $\underline{100310}$

15. Paes MR, Maftum MA. Comunicação entre equipe de enfermagem e pacientes com transtorno mental em um serviço de emergência. Cienc Cuid Saude. [periódico na internet]. 2013 [citado 2017 dez. 20];12(1):55-62. Disponível em: http://periodicos.uem.br/ojs/index.php /CiencCuidSaude/article/view/15830

16. Manias E, Geddes F, Watson B, Jones D, Della P. Perspectives of clinical handover processes: a multi-site survey across different health professionals. J Clin Nurs. [periódico na internet]. 2016 [citado 2017 dez. 20];25(1-2):80-91. Disponível em: https://www.ncbi.nlm.nih.gov/pubmed $\underline{126415923}$

17. Manias E, Watson B. Moving from rhetoric to reality: Patient and family 
involvement in bedside handover. Int $\mathbf{J}$

Nurs Stud. [periódico na internet].

2014 [citado 2017 dez.

20];51(12):1539-41. Disponível em:

http://www.journalofnursingstudies.co m/article/S0020-7489(14)00207-

7/abstract

18. Panesar R, Albert B, Messina $C$, Parker M. The effect of an electronic SBAR communication tool on documentation of acute events in the pediatric intensive care unit. Am J Med Qual. [periódico na internet]. 2016 [citado 2017 dez. 20];31(1):64-8. Disponível em: https://www.ncbi.nlm.nih.gov/pubmed $\underline{125274104}$

Participação dos autores:

Valera IMA trabalhou na concepção teórica, coleta de dados, análise estatística, elaboração e redação final do texto.

Oliveira JLC trabalhou na concepção teórica, análise estatística, elaboração e redação final do texto.

Pereira ACS trabalhou na análise estatística, elaboração e redação final do texto.

Dias AM trabalhou na elaboração e redação final do texto.

Haddad MCFL trabalhou na redação final do texto.

Matsuda LM trabalhou na concepção teórica, análise estatística e redação final do texto.

Recebido: 05.05.2018

Revisado: 08.08.2018

Aprovado: 09.08.2018 